\title{
THE EFFECT OF MINERAL TRIOXIDE AGGREGATE ALONE AND IN COMBINATION WITH BIPHASIC CALCIUM PHOSPHATE ON HEALING OF MANDIBULAR DEFECTS IN RABBIT
}

\author{
Gailan M. Galal ${ }^{1 *} B D S$, Samia S. Abd El Rehim² PhD, Sahar S. Karam ${ }^{3} P h D$
}

\begin{abstract}
INTRODUCTION: Critical sized bone defects above critical size do not heal completely by themselves and thus represent major clinical challenge to reconstructive surgery. Numerous bone substitutes have already been used to promote bone regeneration. Incorporation of bone grafts such as biphasic calcium phosphate (BCP) as osteoconductive materials has been proven to be effective in promoting bone healing in oral surgery. As it is considered a potential candidate for bone regenerating biomolecules which can help in rapid bone repair because of its composition, the biocompatible material "mineral trioxide aggregate"(MTA) is suggested to be promising.

OBJECTIVES: To compare the efficacy of MTA on the process of bone healing alone and the combination of MTA with biphasic calcium phosphate

MATERIALS AND METHODS: Sixteen rabbits will be divided into two experimental groups: (1) MTA, (2) MTA+BCP. Right and left critical size bone defects will be performed in the edentulous area of rabbit mandibles (diastema). Right side bone defects will be filled with MTA, while the left side bone defects will be filled with MTA in combination with BCP. The effect of both MTA and BCP on defect healing will be assessed histologically, histomorphometrically and immunohistochemically after 6 weeks postoperatively.

RESULTS: Greater amount of new bone formation was noticed in the CSDs loaded with MTA and BCP. The amount of new bone formed was significantly higher in the MTA+BCP group when compared with the MTA group. Also bone cells activities appeared greater in the former group.
\end{abstract}

CONCLUSIONS: MTA induces new bone formation via an osteoinductive action and can be added to bone grafts to enhance bone regenerative capacity.

KEYWORDS: Mineral Trioxide Aggregate, Biphasic Calcium phosphate, bone defects, rabbit.

1- Demonstrator of Oral Biology, Faculty of Dentistry, Alexandria University.

2- Professor of Oral Biology, Faculty of Dentistry, Alexandria University.

3- Professor and Head of Oral Biology department, Faculty of Dentistry, Alexandria University.

*Corresponding author:

E-mail: cheerfulangel90@hotmail.com

\section{INTRODUCTION}

Mandibular bone defects in both human beings and animals may be caused by removal of large cystic lesion, by trauma or by infections. The techniques of mandibular reconstruction have represented a challenge to facial surgeons and orthopedists (1).

Critical size defects can be defined as bone defects that do not heal spontaneously during the individual's or the animal's lifetime. Such defects heal by fibrous connective tissue formation and limited bone healing occurring at the margins of the defect (2).

For regeneration to occur, autograft, allograft or xenograft must be applied. The use of autologous grafts for the treatment of defects are the gold standard, because it has both osteoinductive and osteoconductive properties. However, it is not optimal choice because of limitation of supply and the risk of morbidity (3).

Bone allografts are alternatives, but they are expensive and carry the risk of disease transmission and adverse host immune reaction. These problems associated with autografts and allografts have increased the need for synthetic bone graft substitutes (4).

A variety of synthetic bone graft substitutes have been developed over the last 30 years, including hydroxyapatite (HA), alloplastic calcium phosphates, bioactive glass and biphasic calcium phosphate (5). Alloplastic calcium phosphate bone substitutes and tricalcium phosphate have been studied extensively due to their composition, which closely resembles the inorganic phase of bone tissue (6).

Biphasic calcium phosphate (BCP) is considered a safe biomaterial and exhibits high biocompatibility. The BCP consists of particles with an average size of 100-500 $\mu \mathrm{m}$ comprise 60\% hydroxyapatite and 40\% ß-tricalcium phosphate. Compounds based on HA exhibit a low bioabsorption, whereas those based on $§$-TCP are more soluble and can be reabsorbed easily. Thus the association between HA and ß-Tricalcium phosphate generates the biomaterial called "Biphasic calcium phosphate", which is reabsorbable but also contains a more stable segment (HA) that maintains the stability of the graft and the more soluble component (ß-TCP) (7).

However, most synthetic bone grafts have limited capacity to reconstitute bone in large defects because they lack the osteoinductivity and osteogenecity of autologous bone grafts. For these reasons, biologically active molecules have been used to modify bone substitutes so as to enhance their bone forming capacity (8).

One of the potential candidates for bone regenerating biomolecules is the Mineral Trioxide Aggregate (MTA). MTA is a biocompatible material that has different uses. It can be used in direct pulp capping of carious and noncarious pulp exposures of asymptomatic teeth (9). It can 
help in bone repair because its composition allows the rapid adhesion and proliferation of cells on its structure.

There is an evidence that MTA promotes a favorable response in the osseous environment with direct bone apposition. MTA surfaces support osteoblast cell attachment, matrix synthesis and RunX2 expression which are essential for osteogenesis (10).

Immunohistochemical analyses and many experiments have shown that mesenchymal cells, including osteoblasts, express Bone-morphogenetic proteins (BMPs) and their receptors during the formation and fracture repair of bones. These glycoproteins are responsible for bringing osteoprogenitor cells to sites of bone formation and repair.

During the cellular events that regulate bone formation and repair, BMPs induce mesenchymal cell proliferation and differentiation and promote the synthesis of the extracellular matrix. Accordingly, they are involved in the differentiation process where osteoprogenitor cells transform into mature osteoblast cells (11).

The cellular response to BMP depends not only on the expression of the gene but also on the expression and location of the surface transmembrane receptors BMPR types $1 \mathrm{~A}, 1 \mathrm{~B}$ and 2 . Bone-morphogenetic protein receptors (BMPR) are members of the TGF-ß family and bind to two types of serine-threonine kinase receptor, type I and type II receptors. Both type I and type II receptors are required for signal transduction (12).

BMP-2 plays a role in regulating osteogenesis. In vitro studies have revealed that the expression of a constitutively active BMP-2 induced the formation of bone matrix, while the inhibition of BMP-2 completely blocked osteoblast differentiation and mineralized bone formation. These results suggest that BMP-2 is required for osteoblast differentiation and bone formation (13).

In the osseous defect, the signal for the repair or formative process is regulated by the BMP-2, which is involved in the initiation of bone formation (14).

Both BCP and MTA exhibit features that can help in the process of bone formation, however, the literature contains unsatisfactory number of studies evaluating the effect of direct MTA delivery on healing capacity of bone graft materials in regenerative procedures, and this is why the current study had been conducted.

This study aimed to compare the efficacy of Mineral trioxide aggregate (MTA) alone and in combination with biphasic calcium phosphate in the regeneration of osseous defects in rabbit.

\section{MATERIALS AND METHODS}

\section{Experimental animals}

The protocol of this research protocol of this research was approved by the Ethical Committee of the Faculty of Dentistry Alexandria University.

Sixteen skeletally mature male New Zealand white rabbits, each weighing $3 \mathrm{~kg}$, were used in this study. The animals were obtained from the Institute of Medical Research Alexandria University. The animals were supplied a regular diet during the whole experimental period. Rabbits have a diphyodont dentition, characterized by successive development of deciduous and permanent sets of teeth. Deciduous dentition, however, is of no clinical significance and is shed within the first few days after birth.

The dental formula for the permanent dentition in rabbits is as follows: I 2/1 C 0/0 P 3/2 M 3/3.
Rabbits were randomly divided into 2 groups, 8 animals in each group as follows:

Group I: MTA group (8 rabbits)

Group II: MTA+BCP group (8 rabbits)

\section{Materials:}

Mineral trioxide aggregate (MTA) (MTA Cem, NEXOBIO Co, Korea) and Biphasic calcium phosphate (BCP) (DM BONE, META BIOMED Co,Korea ) were purchased.

\section{Surgical procedure:}

General anesthesia was induced by an intramuscular injection of a combination of $25 \mathrm{mg} / \mathrm{kg}$ body weight ketamine hydrochloride (Ketamine® $50 \mathrm{mg} / \mathrm{ml}$ SIGMA TEC CO, Egypt) and $5 \mathrm{mg} / \mathrm{kg}$ body weight Xylazine hydrochloride ( Xyla Ject ${ }^{\circledR}$ ADWIA Co, $10^{\text {th }}$ of Ramadan City , Egypt). The mandible was shaved on both sides and the oral commissures were widened by cutting through the corner of the mouth. Full mucoperiosteal flaps were raised intraorally on both sides of the mandible in each animal in the edentulous region of the alveolar ridges of the body of the mandible (diastema). Critical size osseous defects measuring $3 \mathrm{~mm}$ length $\mathrm{x} 3 \mathrm{~mm}$ width $\mathrm{x} 3 \mathrm{~mm}$ depth were prepared in the edentulous left and right regions of the alveolar ridge of the mandible, using a sterile surgical bur under water cooling system (15).

Right side osseous defects were filled with Mineral trioxide aggregate (MTA), while left side bone defects were filled with MTA and Biphasic calcium phosphate (BCP).

Euthanasia was done after 6 weeks by an overdose of Thipentone Sodium (Glazar Exports Co, Dinshaw Wachha, India) anesthetic solution with rapid injection. The mandibles were dissected out, sectioned into two halves. Serial sagittal sections of $5 \mu \mathrm{m}$ thickness were cut and stained with Hematoxylin \& Eosin.

Immunohistochemical analysis was done to trace the reaction, which was identified as intense, strong, moderate, mild, weak or negative by using anti-morphogenetic protein antibody (Anti-BMP2 antibody(ab6285), ABCAM, United Kingdom).

Histormorphometric analysis was done using image $\mathrm{J}$ software (16) to obtain the percentage of surface area of the formed bone compared to the total surface area of the created bone defect in association with the two variants of the loaded materials in the defect.

\section{Statistical analysis}

The obtained data was statistically analyzed using paired ttest to compare the percentage of the new formed bone in the MTA and MTA+BCP groups.

\section{RESULTS}

\section{I) Histological results}

\section{Group I (MTA group)}

Newly formed bone trabeculae were observed at the lateral wall of the defects directed towards the center. The newly formed bone showed the typical appearance of cancellous variety and exhibited different degrees of maturity ranging from thin to more thick intercommunicating trabeculations (Fig.1).

The line of fusion between the native bone and the newly formed bone was evident and could be clearly seen at the periphery of the defect. A striking density of blood vessels engorged with RBCs could be seen among the bone trabeculae, reflecting a high vascularity to the defect area (Fig.2). 
Osteoblast could be traced at the boundary of new trabeculae which were surrounded by fibrous tissue (Fig.3).

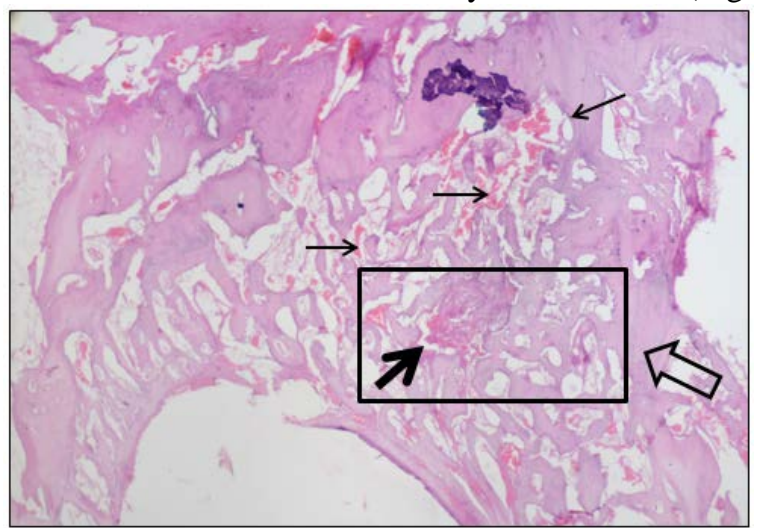

Figure (1): Light photomicrograph (LM) (MTA group, 6 weeks) showing formation of woven bone in the defect surrounded by granulation and fibrous tissue (thick arrow). High vascularity can be observed (thin arrows). Note the line of fusion between native bone and new bone (hollow arrow). H\&E, stain x40.

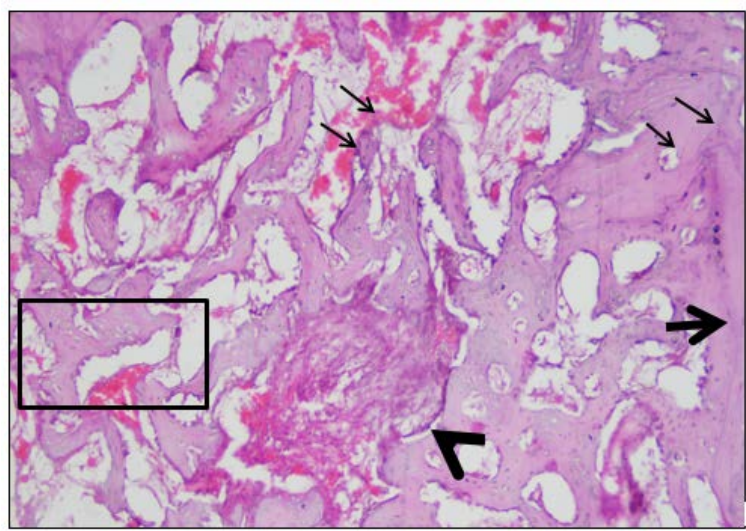

Figure (2): Higher magnification of the inset in the previous figure showing the organization of the bone trabeculae and the line of fusion between the native bone and the newly formed bone (thick arrow). Note the osteoblast density on the bone trabeculae (thin arrows). The center of the defect contains some irregularly organized fibrous tissue (arrowhead). H\&E, stain x100.

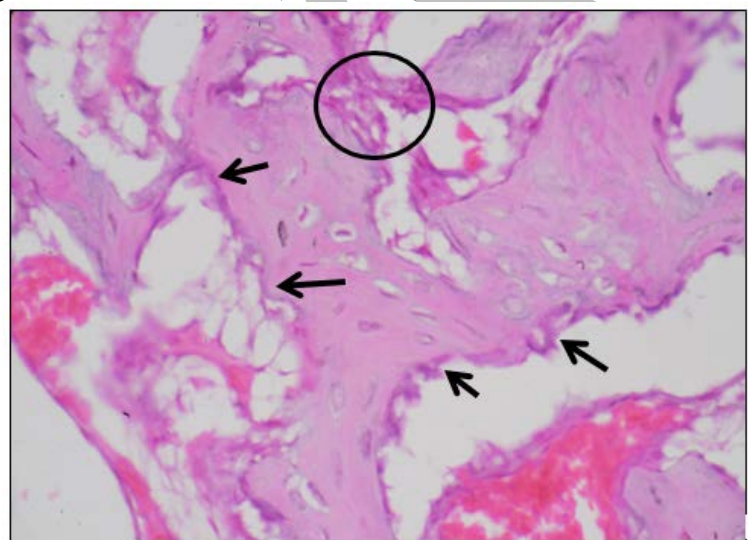

Figure (3): Higher magnification of the black inset showing the intercommunication between new bone trabeculae to coordinate their function lined by numerous osteoblasts cells (arrows). Note the fibrous tissue between the edges which reveals continuation of bone formation bridging spaces between the forming trabeculae (circle). H\&E stain, $\mathrm{x} 400$.

\section{Group II (MTA+BCP)}

There was a greater amount of cancellous bone projecting more towards the center of the defect (Fig.4A).

The bone trabeculae were lined and surrounded by continuous lines of voluminous osteoblast cells and surrounded by capillary rich bone marrow (Fig.4B). This vascularity appeared more than in the previous group.

In some defects, compact bone formation was noted near the central part of the defect with the cancellous variety prevailing at the periphery (Fig.5A). The compact bone consisted of numerous Haversian systems enclosing rich blood supply within their Haversian canals (Fig.5B).

The line of fusion could be traced between the native bone and the newly formed bone, although it seemed more faint and indistinct in many points (Fig.5A, B).

Some osteoclasts were seen within Howship's lacunae indicating an activity of bone resorption (Fig.6).



Figure (4): A: L.M (MTA+BCP, 6 Weeks) showing a greater amount of cancellous bone projecting towards the center of the defect. H\&E, stain, original magnification $x 40$.

B: Higher magnification of the black inset showing the bone trabeculae surrounded by voluminous osteoblasts and capillary rich bone marrow. H\&E, stain $\mathrm{x} 100$.

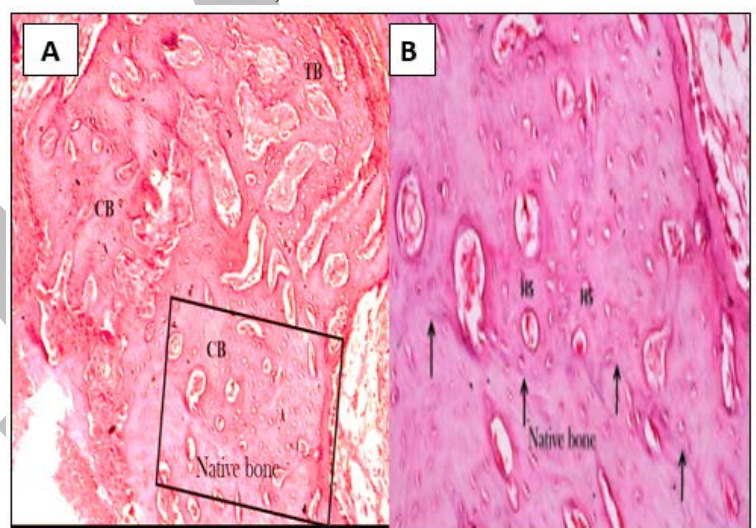

Figure (5): A: LM (MTA+BCP, 6 weeks) showing compact bone formation near the central part of the defect. H\&E, stain x100.

B: Higher magnification of the black inset showing numerous Haversian systems enclosing rich blood supply within their Haversian canals. Note, the line of fusion between the native bone and the newly formed bone (arrows). H\&E stain, x100.

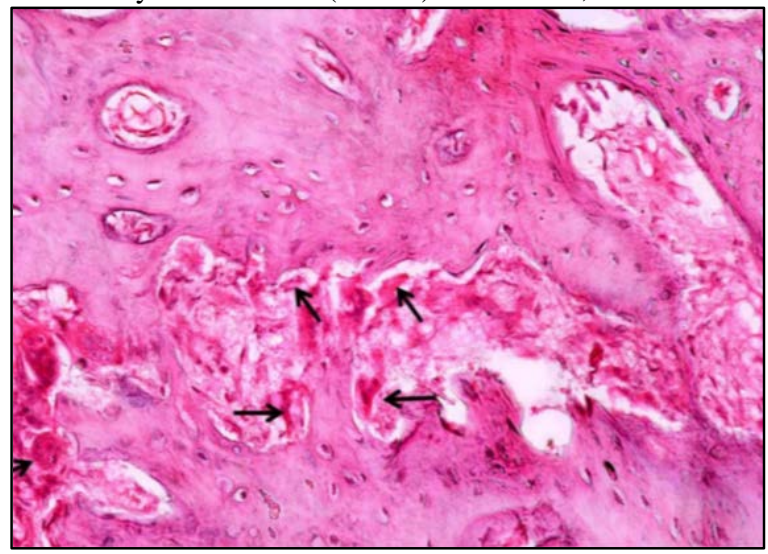


Figure (6): LM (MTA+BCP, 6 weeks) showing an area of active bone resorption. Note the several osteoclast cells residing in Howship’s lacunae. H\&E stain, x400.

\section{II)Histomorphometric analysis}

The mean values of the percentages of bone surface area formed during the healing of the bone defects in the MTA group and the MTA+BCP group after 6 weeks were calculated and demonstrated in table (1).

The table reveals that the amount of the surface area of the newly formed bone is greater in MTA group than in MTA+BCP group and even the difference is significant, revealing a positive effect of the combination of MTA with BCP.

Table (1): Percentage of the surface area of the newly formed bone in the defects

\begin{tabular}{|c|c|c|c|}
\hline Group & $\begin{array}{c}\text { MTA } \\
\text { group }\end{array}$ & $\begin{array}{c}\text { MTA+BCP } \\
\text { group }\end{array}$ & P value \\
\hline Mean \pm SD & $29.76 \pm 4.80$ & $41.77 \pm 7.65$ & $0.01^{*}$ \\
\hline Min.-Max. & $20.3-35.4$ & $30.4-57.8$ & \\
\hline
\end{tabular}

*: Statistical significance $(\mathrm{p} \leq 0.05)$

\section{DISCUSSION}

Mineral Trioxide Aggregate is an endodontic material that was introduced by Mahmoud Torbinejad at Loma Linda University, California, USA. It provides the physical properties, setting requirements for an ideal root repair material. Studies on MTA revealed that it not only exhibits good sealing ability, excellent long term prognosis and good biocompatibility but it favors tissue regeneration as well. MTA consists of tricalcium silicate, tricalcium aluminate, tricalcium oxide, silicate oxide and bismuth oxide.

DM BONE Biphasic calcium phosphate bone graft was the material of choice to act as a delivery vehicle for the MTA because of its highly porous nature of up to $70 \%$ porosity and of pore size 250-1000 $\mu \mathrm{m}$.

Our findings regarding the MTA effect on new bone formation in critical size osseous defects showed that the MTA notably promoted bone formation and exhibited an osteoconductive property.

The new bone formed in the MTA+BCP group was higher, bony trabeculae were thicker and the density of osteocyte content was notably larger than those found in the MTA group. These results are in agreement with the findings of Koh et al (17) who revealed that MTA offers a biologically active substrate for bone cells and stimulates interleukin production. MTA is also said to stimulate cytokine production in human osteoblasts. Their investigation relied on studying the cytomorphology of osteoblasts in the presence of MTA and examined cytokine production by these cells. They concluded that the cells appeared healthy in contact with MTA and that there were raised levels of Interleukins.

In another study performed by Arens and Torabinejad (18) it was proven that osseous repair of furcation perforations could be treated with MTA. MTA showed good interaction with bone-forming cells: cells remained viable and released collagen even after 72 hours with good adherence.

Providing further confirmation to the positive action of the MTA on bone, Nascimento et al (19) evaluated the role of MTA in the bone healing process and wanted to verify if the calcium hydroxide can improve and accelerate this process. A bone defect was created in the mandibles of fourty male wister rats, which were divided into two groups considering or not the use of calcium hydroxide before treatment. The results showed that MTA was able to induce bone regeneration alone and had its action optimized when combined to calcium hydroxide.

Zhu et al (20) examined the adhesion of human osteoblasts to root-end filling materials such as MTA, composite and amalgam by scanning electron microscopy. Root-end filling materials were added to well culture plates and then human osteoblasts were seeded into the wells. After 1 day in culture osteoblasts attached and spread on MTA by forming a monolayer. Few cells were spreading on composite and amalgam which indicated that osteoblasts have a favorable response to MTA compared to amalgam and composite. This observation is thought to confirm the current observation of the outstanding proliferation of osteoblasts which was evident by their voluminous size and intimate attachment on the boundary of the new generated trabeculae.

The current results of the increased activity of osteoblasts are supported by the study of Perinpanayagam et al (21) to determine if MTA support Runx2 expression in osteoblasts which is necessary for periradicular healing. Human alveolar bone cells were grown on alternative formulations of MTA. Cell-surface interactions were visualized by scanning electron microscopy and gene expression was examined by reverse-transcription polymerase chain reaction and Western blot analysis. Results showed that MTA support cell attachment and Runx2 expression in osteoblasts.

The wide range of using MTA has been applied in association with pulp regeneration after capping procedures. Min et al (22) found a higher frequency and thicker reparative hard tissue formed when MTA was used over mechanically exposed pulp compared to calcium hydroxide, they contributed their finding to be due to the stronger immunostaining in active odontoblast-like cells which suggest that MTA has a superior effect on odontoblastic activity. In this study, it has been proven that MTA exhibited regenerative effect than other materials used in different studies and this is thought to support the revealed effect of MTA when used in other locations as used in the current study.

In the current study, MTA+BCP group exhibited new bone formation with dense osteoblast lining which appeared voluminous and active. These results are in agreement with Heziami et al (23) who proved that MTA can up-regulate expression of transcription factors such as RunX2 which support osteoblast cell attachment.

The positive bone balance observed during the healing of the defects suggests that a simple and a direct form of MTA delivery promotes osteoconductive bone formation. Thus, MTA delivery would be promising as a method of achieving rapid and predictable bone regeneration of osseous defects in different locations not only as a root repair material.

\section{CONCLUSION}

MTA with Biphasic calcium phosphate as a carrier may be effective for achieving favorable new bone formation in large critical sized defects as both materials have an osteoconductive property. This approach is expected to 
provide promising clinical outcomes in other fields of dentistry.

\section{CONFLICT OF INTEREST}

The authors declare that they have no conflicts of interest.

\section{REFERENCES}

1. GOMES, C., et al. Bone regeneration in mandible defect with autograft bone and cell suspension from bone marrow in rabbits. Arq. Bras. Med. Vet. Zootec. 2011, 63.4: 836-843.

2.EZIRGANLI, Şeref, et al. The effect of local simvastatin application on critical size defects in the diabetic rats. Clin Oral Implants Res. 2014, 25.8: 969-976.

3. Jang ES,Park JW,Kweon H,Lee KG,Kang SW,Baek DH,et al.Restortion of peri-implant defects in immediate implant installations by Choukroun platelet-rich fibrin and silk fibrin powder combination gaft. Oral Surg Oral Med Oral Pathol Oral Radiol Endod. 2010;109(6):831-6.

4. Moore WR,Graves SE,Bain GI.Synthetic bone graft substitutes. ANZ J Surg. 2001;71(6):354-61.

5. Giannoudis PV,Dinopoulos H,Tsiridis E.Bone substitutes: an update. Injury. 2005;36(3):S20-S27.

6. JENSEN, Simon Storgård, et al. Evaluation of a novel biphasic calcium phosphate in standardized bone defects. A histologic and histomorphometric study in the mandibles of minipigs. Clin Oral Implants Res. 2007, 18.6: 752-760.

7. KIM, S. H., et al. Histological and immunohistochemical evaluation of biphasic calcium phosphate and a mineral trioxide aggregate for bone healing in rat calvaria. Int. J. Oral Maxillofac. Surg. 2015, 44.4: 535-542.

8. Xu L,Lv K,Zhang W,Zhang X,Jiang X,Zhang F. The healing of critical-size calvarial bone defects in rat with rhPDGF-BB,BMSCs, and ß-TCP scffolds. J. Mater. Sci. Mater. Med. 2012;23(4):1073-84.

9. Komabyashi T,Zhu Q.Innovative endodontic therapy for anti-inflammatory direct pulp capping of permanent teeth with a mature apex. Oral Surg Oral Med Oral Pathol Oral Radiol Endod. 2010;109;75-81.

10. PINHEIRO, Antonio LB, et al. Does LED phototherapy influence the repair of bone defects grafted with MTA, bone morphogenetic proteins, and guided bone regeneration? A description of the repair process on rodents. Lasers Med. Sci. 2012, 27.5: 1013-1024.

11.WOZNEY, John M.; ROSEN, Vicki. Bone morphogenetic protein and bone morphogenetic protein gene family in bone formation and repair. Clin. Orthop Relat. Res. 1998, 346: 26-37.

12. CHEN, D., et al. Differential roles for bone morphogenetic protein (BMP) receptor type IB and IA in differentiation and specification of mesenchymal precursor cells to osteoblast and adipocyte lineages. J. Cell Biol. 1998, 142.1: 295-305.

13. LIN, Shuxian, et al. The biological function of type I receptors of bone morphogenetic protein in bone. Bone Res. 2016, 4: 16005.

14. ONISHI, T., et al. Distinct and overlapping patterns of localization of bone morphogenetic protein (BMP) family members and a BMP type II receptor during fracture healing in rats. Bone Res. 1998, 22.6: 605-612.

15. Eleftheriadis E, Leventis MD, Tosios KI, Faratzis G, Titsinidis S, Eleftheriadi I, et al. Osteogenic activitiy of BETA-tricalcium phosphate in a hydroxyl sulphate matrix and demineralized bone matrix: a histological study in rabbit mandible. J Oral Sci 2010;52(3):377-84
16. Yugoshi LI, Sala MA, Brentegani LG, Carvalho TLL. Histometric study of socket healing after tooth extraction in rats treated with diclofenac. Braz Dent J. 2002;13(2):92-6.

17. Koh E, McDonald F, Pitt Ford T, Torabinejad M. Cellular response to mineral trioxide aggregate. J Endod. 1998;24: 543-547.

18. Arens DE, Torabinejad M. Repair of furcal perforation with mineral trioxide aggregate. Oral Surg Oral Med Oral Pathol Oral Radiol Endod. 1996;82: 84-88.

19. Do Nascimento, Cássio, et al. Bone repair using mineral trioxide aggregate combined to a material carrier, associated or not with calcium hydroxide in bone defects. Micron. 2008;39.7: 868-874.

20.Zhu, Qiang, et al. Adhesion of human osteoblasts on rootend filling materials. J Endod. 2000; 26.7: 404-406.

21.PERINPANAYAGAM, Hiran; AL-RABEAH, Ebtehal. Osteoblasts interact with MTA surfaces and express Runx2. Oral Surg Oral Med Oral Pathol Oral Radiol Endod. 2009;107.4: 590-596.

22. Min K, Park H, Lee S, Park S, Hong C, Kim H, et al. Effect of Mineral Trioxide Aggregate on Dentin Bridge Formation and Expression of Dentin Sialoprotein and Heme Oxygenase-1 in Human Dental Pulp. J Endod. 2008: 106: 609-14.

23. Al-Hezaimi K, Salameh Z, Al-Fouzan K, Al Rejaie M, Tay F. Histomorphometric and Micro-computed Tomography Analysis of Pulpal Response to three Different Pulp Capping Materials. J Endod. 2011; 37:507-12. 